http://jmscr.igmpublication.org/home/

ISSN (e)-2347-176x ISSN (p) 2455-0450

crossref DOI: https://dx.doi.org/10.18535/jmscr/v8i5.75

Journal Of Medical Science And Clinical Research

\title{
Guttate Morphea-A Rare Localised Scleroderma Variant
}

\author{
Authors \\ Dr Pooja $\mathbf{K}^{1^{*}}$, Dr M.S.Srinivasan ${ }^{2}$ \\ ${ }^{1}$ Junior Resident, ${ }^{2} \mathrm{Head}$ of the Department \\ Department of Dermatology, Venereology and Leprosy, \\ Chettinad Hospital and Research Institute, Kelambakkam, India \\ *Corresponding Author \\ Dr Pooja K \\ Junior Resident, Department of Dermatology, Venereology and Leprosy, \\ Chettinad Hospital and Research Institute, Kelambakkam, India

Abstract
Morphea is an autoimmune disease localized to the skin. It is also known as localized scleroderma.
Scleroderma is a multisystem connective tissue disorder characterized by thickening and hardening of skin
with loss of elasticity. In our case, we represent a 34 year-old male with guttate morphea. The clinical
presentation, examination findings and histopathology confirmed it as guttate morphea.
Keywords: Autoimmune disease, localized scleroderma, Guttate morphea.

\section{Introduction}

Morphea which is also known as localized scleroderma is a chronic autoimmune connective tissue disorder due to varied etiology such as trauma, viruses, borrelia, drugs such as pentazocine, paclitaxel, etc. Guttate morhea is one of the rarest sub types, and it represent less than $1 \%$ of the cases worldwide. Depending on the severity and type of morphea, underlying structures such as muscles, fascia, bones can be affected.

There are many types of morphea. Based on Peterson et al, morphea can be classified as

Plaque morphea

Generalized morphea

Bullous morphea

Linear morphea

Deep morphea
Various genetic factors, environmental factors, autoimmunity are involved in the pathogenesis of the disease including TGF-b, MMP, growth factors.

\section{Case Report}

A 15 year old male patient presented with c/o multiple asymptomatic depressed lesions over both legs, forearms, face for past 1 year.

There was initially appearance of a single depressed lesion over the left leg, followed by appearance of multiple new asymptomatic lesions over both legs, hands, face.

There were no scales and no change in color with sun exposure, not tender, there is no bleeding or discharge, not preceded by erythema, in the last one year the lesion increased gradually in number and involved the back and shoulders, but no changes in the sizes or color of individual lesions. 
History of appearance of new lesions present.

No history of any trauma.

No history of koebnerization.

No history of exposure to any drugs or chemicals.

No known comorbidities.

No significant family history. No history of smoking or alcoholism or drug intake. Sleep, appetite regular.

Lesions were not associated with any systemic symptom.

\section{Cutaneous Examination}

Patient is conscious and oriented, moderately built.

Detailed cutaneous examination revealed multiple $0.5 \mathrm{~cm}-1 \mathrm{~cm}$ hypopigmented, well defined, depressed macules present over extensor aspect of shins of both legs, back, distal end of forearms, forehead, dorsum of right foot, palms.

A single lesion over the face was also present.

No scales or erythema was present.

Nails: normal.

Genitalia: normal.

Scalp: normal.

Oral mucus membrane: normal.

\section{Laboratory Investigations}

Complete blood cell count and biochemistry results were under normal limits. Anti ss DNA, Anti scl-70 and ANA antibodies were normal.

\section{Histopathology}

A 5.0mm Skin punch biopsy was taken from the Right calf muscle of lower limb. Histopathological examination shows focal thinning of the epidermis, there is increase in dermal collagen with compression of adnexal structures.

The mid dermis and the deep dermis show thick collagen bundles arranged haphazardly. No atypia or malignancy.

\section{Diagnosis}

The clinical findings, examination \& histopathology confirms GUTTATE MORPHEA

\section{Differential Diagnosis}

Anetoderma,

Guttate morphea,

Atrophoderma,

Lichen sclerosus,

Lipoid Proteinosis,

Cutaneous Atrophy.

\section{Treatment}

Topical Tacrolimus $0.1 \%$ ointment OD, Clobetasol propionate $0.005 \%$ +calcipotriol $0.05 \%$ ointment hs,

T. Methylprednisolone 4mg OD for 14 days was initially given as treatment. $\mathrm{Pt}$ asked to come for follow up every 2 weeks.

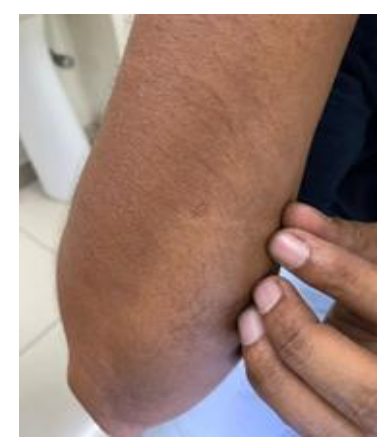

Fig 01

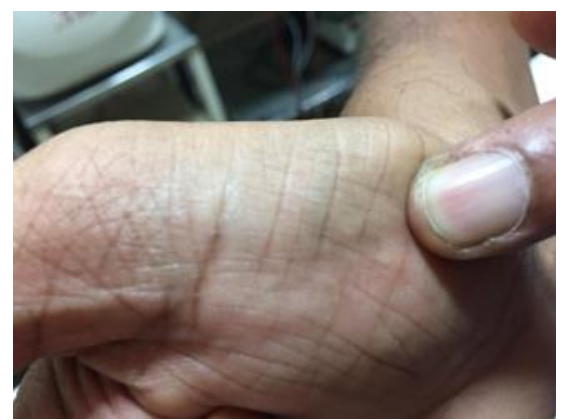

Fig 02

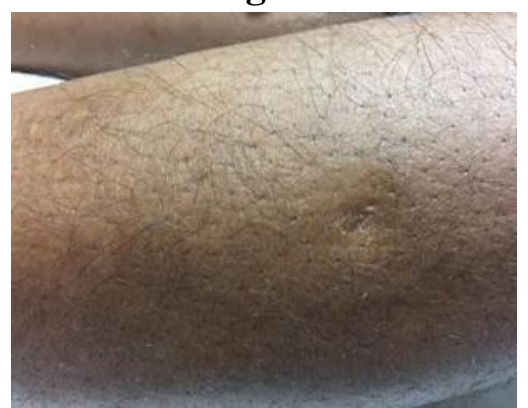

Fig 03 


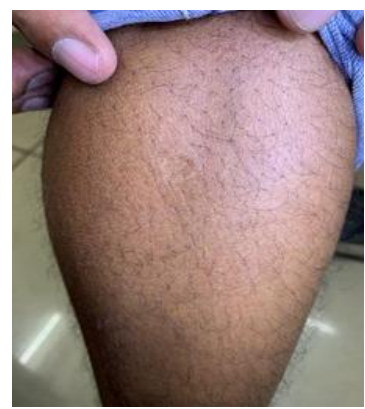

Fig 04

Fig. 1 to Fig. 4: multiple, hypopigmented papules ranging from $0.5 \mathrm{~mm}$ to $1 \mathrm{~mm}$ on the hands and legs of the patient

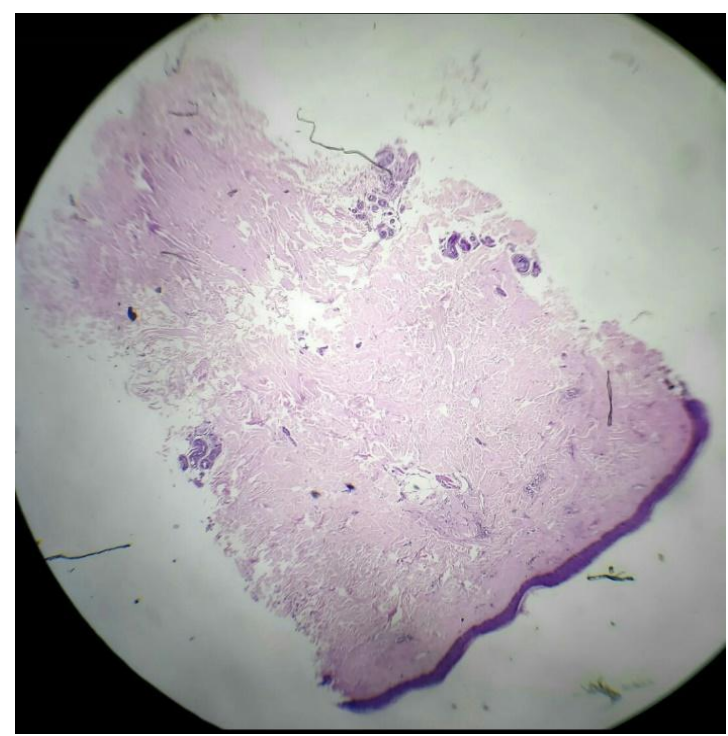

Fig 05

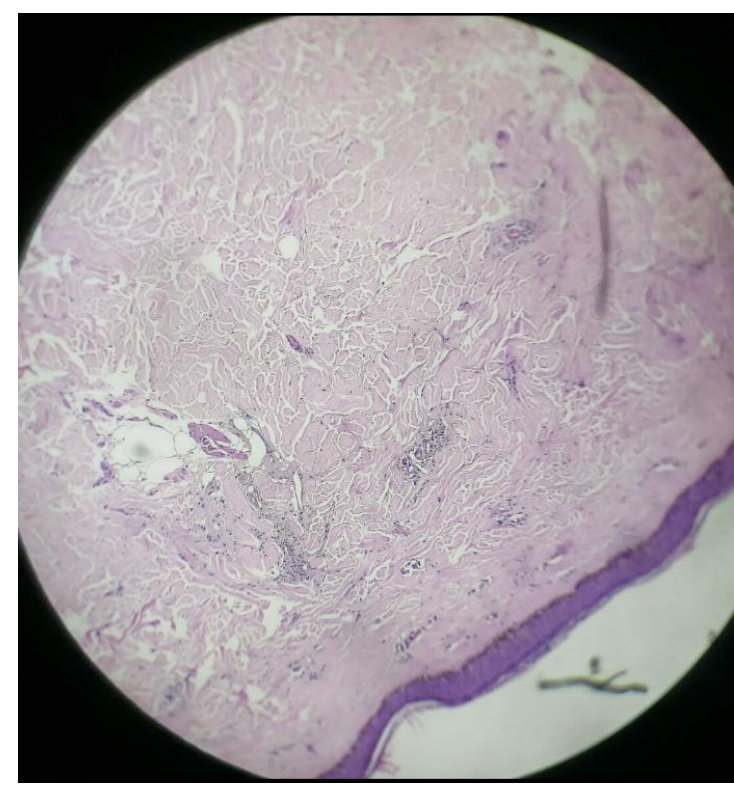

Fig 06

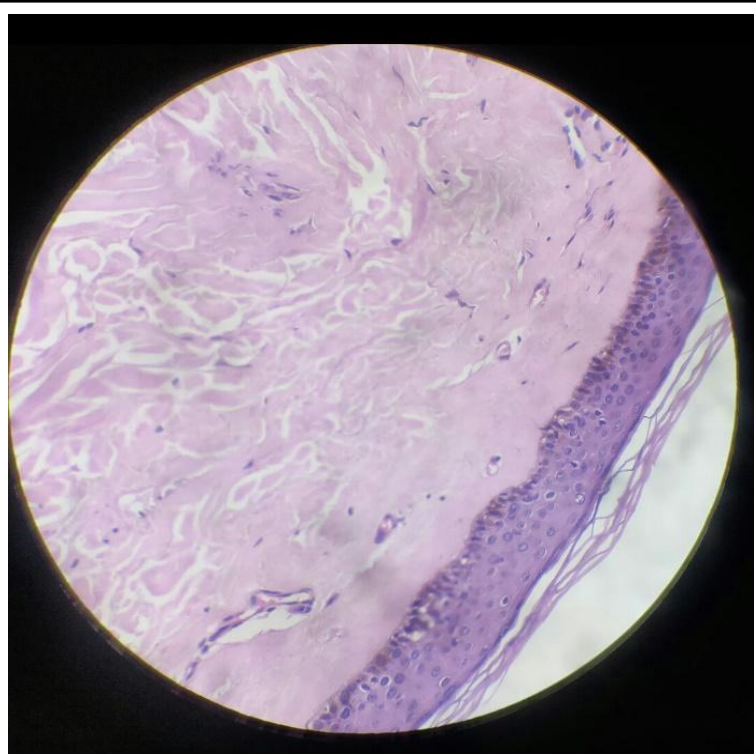

Fig 07

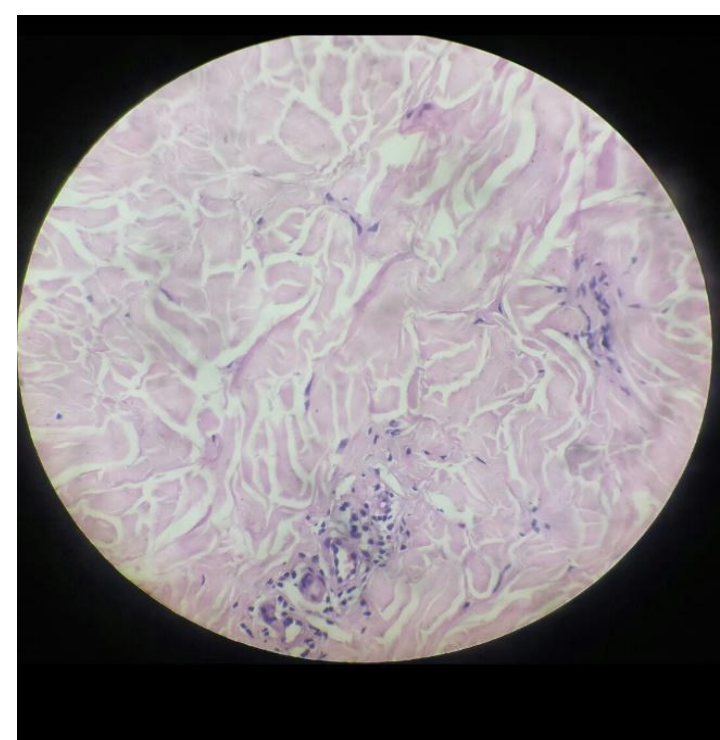

Fig 08

Fig 5 to Fig 8: Thick collagen bundles are present in dermis along with atrophy of adnexal structures

\section{Discussion}

Morphea is an autoimmune disorder. It affects women three times more than men. It is a rare condition with an incidence of 2.7 per million. Various etiologies such as rasiation, silicone impants, trauma, viral infections, drugs have been reported. Increased synthesis of collagen \& decreased collagenase, increased matrix metalloproteinases, TGF-b are depicted in the pathogenesis.

Morphea resembles lichen sclerosus clinically but can be easily differentiated by the fact that 
morphea is a localized type of scleroderma, and it is characterized by thickened and hardened skin with histopathology of thickened collagen bundles $\&$ atrophy of adnexal structures. Lichen sclerosus reveals thinning of epidermis, follicular \& perifollicular plugging, hyperkeratosis, with lymphocytic infiltrate.

Guttate Morphea is nothing but plaque type of morphea which are small, with sharply demarcated well defined borders and chalky white macules \& patches. There are three stages: ivory/chalk white, scaly, and atrophic stages.A typical plaque of morphea presents with oval shaped hypo or hyperpigmented indurated patches with ivory or chalky white colour surrounded by a lilac halo or ring. Generalized cases with involvement of more than two sites can present with other complaints such as dysphagia, dyspnea or Raynaud's phenomenon.

ANA, Anti scl-70 antibodies \& anti ss DNA antibodies can be elevated in these patients. There is thickened collagen bundles in the dermis and subcutis, vessel wall thickening \& narrowed lumen \& compression and atrophy of adnexal structures along with thinning of epidermis.

Treatment options include topical corticosteroide, tacrolimus and calcipotriol in topical therapy. Depending on the severity, PUVA or UVB, oral steroids, methotrexate, hydroxychloroquine or mycofenolate mofetil can be given.

\section{References}

1. Wedad Khana, Rasha Alasiri, Hadeel Alkhatabi. GUTTATE MORPHEA:A CASE REPORT. Int $\mathrm{J}$ Biol Med Res. 2016;7(3):5669-5671

2. Nicholas A Ross, MD. Jason B Lee, MD. Matthew s. Keller, MD. Atrophodrema like Guttate Morphea. www.MDedge.com/Cutis.2018; Volume 102(2):E7-10. 